

\section{MEMBERS AND SUBSCRIBERS}

T 1 HE Instituto Internacional de Literatura Iberoamericana was organized in 1938 in order to advance the study of Iberoamerican Literature, and to intensify cultural relations among the peoples of the Americas.

To this end, the Institute publishes the REVISTA IBEROAMERICANA, on the 15 th day of the months of February, June and October of each year and it maintains Standing Committees to facilitate: the coordination of linguistic and literary research; the promotion of cultural relations; the creation of chairs of Iberoamerican Literature in the United States, and of chairs of North American Literature in Iberoamerica; and the printing of notable books by Iberoamerican authors -in their original languages and in English translation-, and of works of erudition and text books for teaching.

Members of the Institute meet every two or three years, and are of two categories: regular members who pay $\$ 4.00$ a year, and Patron Members who pay a minimum of $\$ 10.00$ a year.

Institutions such as universities, colleges and libraries will become subscribers (at $\$ 4.00$ a year), or Subscribing Patrons (at a minimum of $\$ 10.00$ a year) without holding membership in either case.

Regular members and suscribers receive the incoming issues of the REVISTA IBEROAMERICANA free, but Patrons (whether Members or Subscribers) receive in addition all the incoming publications of the Institute, such as the CLASICOS DE AMERICA, the MEMORIAS of the Congresses, etc., and their names will be printed in the REVISTA IBEROAMERICANA at the end of the year.

\section{NOT I C E}

We hope that you will become a member of the Institute, and if you cannot become one of its Patrons we urge that you obtain a Patron Subscription for your school library, which then will receive the full cultural benefit of our publications. Let us count upon your cooperation.

Name of regular member or subscriber $(\$ 4.00) \ldots \ldots \ldots \ldots \ldots \ldots \ldots$

Name of Patron Member or Subscriber $(\$ 10.00$, minimum $) \ldots \ldots \ldots \ldots$

Address in full

Please make your checks payable to the Instituto Internacional de Literatura Iberoamericana and mail your dues to Dr. Marshall R. Nason, Treasurer -University of New Mexico, N. M.-, the only person with whom you are to deal in matters relating to the circulation and distribution of all the publications of the Institute. 


\section{SOCIOS Y SUSCRITORES}

E

L. Instituto Internacional de Literatura Iberoamericana se organizó en 1938 con el fin de adelantar el estudio de la Literatura Iberoamericana, e intensificar las relaciones culturales entre todos los pueblos de América.

Con este fin, el Instituto publica la REVISTA IBEROAMERICANA, cada cuatro meses, en los de febrero, junio y octubre, y mantiene Comisiones Permanentes encargadas de facilitar: la coordinación de investigaciones lingüísticas y literarias; el intercambio cultural; la creación de cátedras de Literatura Iberoamericana en los Estados Unidos, y la de cátedras de Literatura Angloamericana en Iberoamérica; y la publicación de obras notables de autores iberoamericanos -en el idioma original y en traducción inglesa-, y la de obras de erudición y textos de enseñanza.

Los socios del Instituto se reúnen en Congresos cada dos o tres años, y son de dos categorías: el socio de número, cuya cuota anual es de cuatro dólares en los Estados Unidos y de sólo dos dólares en los demás paises; y el Socio Protector, cuya cuota mínima es de diez dólares al año.

Las bibliotecas, colegios, universidades y demás instituciones que, sin ser socios, sí favorecen al Instituto, son de dos categorías: el suscritor corriente, cuya cuota anual es de cuatro dólares en los Estados Unidos y de sólo dos dólares en los demás países; y el Suscritor Protector, cuya cuota mínima es de diez dólares al año.

La REVISTA IBEROAMERICANA se sirve gratuitamente a los socios de número y a los suscritores corrientes del Instituto, pero tanto los Socios Protectores como los Suscritores Protectores reciben, además de la revista, las demás publicaciones que vayan saliendo, tales como los CLASICOS DE AMERICA y las MEMORIAS, y sus nombres se publican en la REVISTA IBEROAMERICANA al fin de cada año.

\section{A D VERTENCIA}

E1 Instituto invita encarecidamente a quienes simpaticen con los fines que persigue, a que se hagan cuanto antes, ora socios, ora Protectores de él. Quienes así lo apoyen deben enviar su cuota antual, por adelantado, en forma de giro postal o bancario pagadero al Instituto Internacional de Literatura Iberoamericana y por conducto del Dr. Marshall R. Nason, Secretario-Tesorero -University of New Mexico, N. M.-, que es la úmica persona encargada de la circulación y la distribución de las publicaciones del Instituto.

La REVISTA IBEROAMERICANA establecerá el canje con otras publicaciones análogas cuando así lo soliciten por escrito, y siempre y cuando el canje se haga por el conducto único de su Director Literario, Dr. Jullio Jiménea Rueda, Puebla 394, México, D. F. 


\title{
Revista llberoamericana
}

\author{
Organo del Instituto Internacional \\ de \\ Literatura Iberoamericana
}

\author{
Publicación a cargo de: \\ Julio Jiménez Rueda: Director Literario \\ Puebla N 394, México, D. F. \\ Francisco Monterde: Director Técnico \\ Universidad Nacional de México, México, D. F.
}

\section{Coeditores:}

John E. Englekirk

Tulane University

New Orleans, La.

\section{Manuel Pedro González}

University of California,

Los Angeles, Calif.
Sturgis E. Leavitt University of North Carolina Chapel Hill, N. C.

E. Herman Hespelt

(Sección de Anuncios)

New York University

New York, N. Y. 


\section{Mesa Directiva del Instituto Internacional. DE Literatura Iberoamericana}

PRESIDENTE

Ernest R. Moore, Syracuse University, Syracuse, New York VICEPRESIDENTES

Albert R. Lopes, University of New Mexico, Albuquerque, N. M. Gastón Figueira, Montevideo, Uruguay Mariano Picón-Salas, Bogotá, Colombia SECRETARTO - TESORERO

Marshall R. Nason, University of New Mexico, N. M. DIRECTOR DE PUBLICACIONES

Julio Jiménez Rueda

Puebla 394, México, D. F. DELEGADOS

Miguel N. Lira, México; L. E. Nieto Caballero, Colombia; Arturo Uslar Pietri, Venezuela; Augusto Arias y Abel Romeo Castilla, Ecuador; Estuardo Núñez, Perú; Fernando Díez de Medina, Bolivia; Alberto Zum Felde, Uruguay; Cecilia Meireles y William Berrien, Brasil; Raimundo Lida, Argentina; Raúl Silva Castro, Chile; David Vela, Nicaragua; Catalino Arrocha, Panamá.

\section{COMISIONES PERMANENTES}

I. Sección de Coordinación de Investigaciones Lingüísticas y Literarias: Presidente: E. K. Mapes, State University of Iowa, Iowa City, Ia. Vocales: L. B. Kiddle, Julio Jiménez Rueda, Eduardo Neale Silva, Raúl Silva Castro.

II. Sección de Bibliografías:

Presidente: Ernest R. Moore, Syracuse University, Syracuse, New York, Vocales: Madaline Nichols, Ralph Warner, Fermín Peraza Sarausa.

III. Sección General de Publicaciones:

Director: Julio Jiménez Rueda, Puebla 394, México, D. F. Vocales: Sturgis E. Leavitt, Angel Flores, L. B. Kiddle, John E. Englekirk. SUBCOMISIONES

Revista Iberoamericana, Julio Jiménez Rueda, Director Literario. Clásicos de América, Julio Jiménez Rueda, Editor; Coeditores, Arturo Torres-Rioseco, Carlos García-Prada, William Berrien y Mariano Picón-Salas.

Obras de Altos Estudios Literarios y Lingüísticos, Editor, Sturgis E. Leavitt; Coeditores, Otis H. Green, Irving Leonard y Astrojildo Pereira.

Traducciones: Angel Flores, Editor; Coeditores: Harriet de Onís, Katherine Anne Porter, Duddley Poore y G. W. Umphrey.

Diccionarios: Editor, L. B. Kiddle.

IV. Sección de Intercambio Cultural:

Presidente: John A. Crow, University of California, Los Angeles, Cal. Vocales: Lawrence Dugan, Concha Romero James, Albert R. Lopes y William Berrien. 
Esta Revista aspira a constituir, gradualmente, una vital representación de los grandes valores espirituales de la creciente cultura iberoamericana.

Sus directores, así como el Instituto, quieren hacer vivo el lema que cifra el ideal de su obra: A LA FRATERNIDAD POR LA CULTURA.

Se reflejará en sus páginas una clara imagen del pensamiento de Iberoamérica. 
